\title{
Desmoplastic/Nodular Medulloblastoma
}

National Cancer Institute

\section{Source}

National Cancer Institute. Desmoplastic/Nodular Medulloblastoma. NCI Thesaurus. Code C4956.

A medulloblastoma characterized by the presence of nodular, collagenous areas which do not contain reticulin, surrounded by hypercellular areas which contain an intercellular reticulin fiber network. 